\title{
Artropofauna de importancia forense en un cadáver de cerdo en el Callao, Perú
}

\author{
José lannacone 1 \\ ${ }_{1}^{1}$ Laboratorio de Ecofisiología, Facultad de Ciencias Naturales y Matemáticas, Universidad Nacional Federico Villarreal. Calle \\ San Marcos 383, Pueblo Libre, Lima 21, Perú. E-mail: joselorena@terra.com, joseiannacone@hotmail.com
}

\begin{abstract}
Arthropofauna of forensic importance in pig carcass in Callao, Peru. This is the first report of an ongoing study on arthropofauna of forensic importance in Callao, Peru using a baby pig (Sus scrofa Linneaus, 1758) on land as a model to determine the arthropofauna over 84 days of weekly survey between 17 July and 02 October 2 000. A total of 4,405 specimens were collected belonging to five orders and eight families: Cochliomyia macellaria (Fabricius, 1775) (Diptera, Calliphoridae) (81.62\%); Dermestes maculatus (De Geer, 1774) (Coleoptera, Dermestidae) (16.35\%); Fannia canicularis (Linnaeus, 1761) (Diptera, Muscidae) $(0.04 \%)$; Saprinus aeneus (Fabricius, 1775) (Coleoptera, Histeridae) (1.48\%); Necrobia rufipes (De Geer, 1775) (Coleoptera, Cleridae) $(0.45 \%)$; Linepithema humile (Mayr, 1868) (Hymenoptera, Formicidae) (0.02\%); Porcellio Iaevis Latreille, 1804 (Isopoda, Porcellionidae) $(0.02 \%)$ and Hadruroides Iunatus (L. Koch, 1867$)$ (Scorpionida, Iuridae) $(0.02 \%)$. Larvae accounted for $76 \%$, pupae $14 \%$ and adults $10 \%$ of the total collected. The arthropods were into three: necrophages (98.01\%), predators $(1.95 \%)$ and omnivorous $(0.04 \%)$. C. macellaria were significantly higher during the decayed stage; by contrast $D$. maculatus was much higher in dry remains stage. The highest diversity with the Shannon-Weaver $\left(\mathrm{H}^{\prime}\right)$ and Pielou (J) index were found during the advanced decayed stage. The absence of species of genus Chrysomyia RobineauDesvoidy, 1830 is discussed.
\end{abstract}

KEY WORDS. Calliphoridae, Coleoptera, Diptera, forensic entomology.

Los artrópodos están usualmente entre los primeros y más importantes invertebrados que colonizan un cadáver animal y humano (CATTS \& GofF 1992, AMENDT et al. 2000), y siguen una secuencia de sucesión predecible en carcasas animales (TOMBERLIN \& ADler 1998). Mediante la identificación de los insectos presentes y sus estadios de vida, es posible estimar cuanto tiempo el cuerpo ha estado muerto y donde ocurrió la muerte del cadáver (BENECKE 1998). Pudiendo así determinar el intervalo Post Mordem (IPM) (Komar \& BeatTie 1998). Los más importantes grupos de insectos que se alimentan de animales muertos son las moscas Calliphoridae y los escarabajos necrófagos Dermestidae y Silphidae (Souza \& Linhares 1997). La familia Calliphoridae usualmente ovipone a partir de las pocas horas de muerte del cadáver (Hegazi et al. 1991, Byrd \& Butler 1996, Lee 1996, Malgorn \& Coquoz 1999). La artropofauna en un cadáver está ligada a los cambios naturales que tienen lugar en un cuerpo muerto y también a las condiciones ambientales (INTRONA et al. 1998, von ZuBEn et al. 1998, van LAERHOVEn \& ANDERson 1999, Carvalho et al. 2000, Barreto et al. 2002).

La validez de los datos de línea de base empleando los cálculos obtenidos de estudios de descomposición, depende de la clase de modelo no humano empleado. Los cerdos domés- ticos (Sus scrofa (Linneaus, 1758)) parecen ser los animales más aceptables como modelos no humanos para el cálculo del IPM (CATTS \& Goff 1992).

BARRETO et al. (2002) señala que la información con relación a los insectos en cadáveres animales y humanos en Sudamérica es escasa. Además, se ha estudiado más la migración de las especies introducidas del complejo Chrysomyia spp. en Brasil y Costa Rica hacia el norte, que sus migraciones al sur de Sudamérica (BAUMGARTNER 1993). En la literatura principalmente del Brasil, se considera que estas especies han ocasionado un decline repentino poblacional en una especie ecológicamente similar, Cochliomyia macellaria (Fabricius, 1775), nativa de América (Godoy et al. 2002). Además, hay fuertes evidencias que muestran que Chrysomyia hrysomyia Robineau-Desvoidy, 1830 preda sobre Cochliomyia macellaria durante la fase larval (Faria \& Godoy 2001). En el Perú, no se tiene ningún registro publicado de la artropofauna de importancia forense presente en carcasas animales.

De esta forma el objetivo principal de la presente investigación fue determinar la artropofauna de potencial forense, empleando como carcasa a un lechón de Cerdo en Ventanilla, Callao, Perú durante la fase invernal del 2000.

Revista Brasileira de Zoologia 20 (1): 85-90, março 2003 


\section{MATERIAL Y MÉTODOS}

La parte experimental se realizó en el Fundo Oquendo, propiedad de la Universidad Nacional Federico Villarreal, $\mathrm{Km}$ 8, Ventanilla, Callao $\left(11^{\circ} 52^{\prime} \mathrm{S}, 77^{\circ} 07^{\prime} \mathrm{W}\right)$. Esta área fue seleccionada por presentar un amplio terreno cercado con ausencia de cobertura vegetal a $50 \mathrm{~m}$ a la redonda y poca perturbación antrópica. A esa distancia se localizaban algunos cultivos de tomate y camote. La altitud del área de estudio fue de $55 \mathrm{msnm}$, pendiente de $2 \%$ y escasos $\mathrm{mm}$ de precipitación anual (IANnacone et al. 2000a). Según Brack (1986) pertenece a la ecorregión del Desierto Pacífico Costero.

Se utilizó como modelo un ejemplar de lechón de $S$. scrofa "Cerdo" de 45 días de edad y de 3648 g de peso. Este ejemplar fue obtenido de un criadero de cerdos denominado Parque Porcino, localizado en la ribera del Río Chillón-Ventanilla, Callao. El cerdo fue sacrificado utilizando una dosis de $0,71 \mathrm{~mL}$ $\mathrm{Kg}^{-1}$ de succina-colina. El cerdo fue colocado en posición lateral, sobre una malla de acero de abertura de $2 \mathrm{~cm}$ y de área de 70 $\mathrm{cm}$ de largo (L) x $50 \mathrm{~cm}$ de ancho (A), debajo de la cual fue colocado un plástico blanco de $80 \mathrm{~cm}$ x $60 \mathrm{~cm}$. Luego se colocó dentro de una jaula hecha con el mismo tipo de malla con las siguientes dimensiones $70 \mathrm{~cm}$ de Alto x $70 \mathrm{~cm}$ de Largo x $50 \mathrm{~cm}$ de Ancho. La jaula se sujetó con la ayuda de cuatro estacas de madera y se colocó un pequeño cobertor de plástico negro de área de $70 \mathrm{~cm}$ x $50 \mathrm{~cm}$ para proteger a la carcasa del pasaje directo de la luz del sol.

La instalación del experimento con el cadáver de cerdo se realizó el 10 de julio del 2000 . Se realizaron un total de 12 evaluaciones semanales. Los muestreos se realizaron entre $10 \mathrm{am}$ a 12 am del 17 de julio al 02 de octubre del 2000 (fase invernal). El experimento duró 84 días, hasta que la entera carcasa fue consumida. La artropofauna, tanto de adultos como de inmaduros fue colectada con la ayuda de pinzas entomológicas y redes aéreas. Se realizó un conteo y colecta total de la fauna cadavérica presente en cada fecha evaluada. Sin embargo, para evitar la perturbación en la siguiente colecta semanal, en especial las formas larvarias de insectos presentes, luego de su verificación morfoespecífica, se devolvió a la carcasa gran cantidad del material colectado vivo. Posteriormente a las formas larvarias no devueltas a la carcasa y mantenidas en el laboratorio se les colocó en pequeños viales de vidrio y se preservó en alcohol al 70\% glicerinado y a las formas adultas con torundas de algodón empapado con cloroformo y luego fueron montados en alfiler en cajas entomológicas, siguiendo las recomendaciones propuestas por PeÑa (1992), a excepción de los crustáceos y arácnidos. Inicialmente, las especies insectiles fueron identificadas a nivel del taxón familia con la ayuda de las claves taxonómicas de BOrror et al. (1989) y LiU \& Greenburg (1989). Para identificar los adultos de Cochliomyia macellaria y diferenciarlos principalmente del complejo Chrysomyia spp. se utilizó las claves de Dale (1985), Dear (1985) y Tantawi \& El Kady (1997) y para el caso de las especies de Coleóptera se recurrió a especialistas y a las claves para Cleridae de Solervicens (2001). Para identificar los alacranes se empleó las claves propuestas por Stockwell (1992). Para la identidad de los tres estadios larvales y de la pupa de C. macellaria y diferenciarlos de otras especies de Calliphoridae; y para identificar a la larva de Dermestidae se emplearon los caracteres morfológicos propuestos por GREenberg \& SzYSKa (1984), Liu \& GREENBERG (1991) y AMORIN \& Ribeiro (2001). Las especies fueron designadas en cada una de las tres categorías ecológicas según su rol trófico: necrófagas, omnívoros y depredadores según CatTs \& Goff (1992). Material representativo de las especies adultas e inmaduras de insectos fue depositado en el Museo de Entomología, Programa Nacional de Control Biológico, Servicio Nacional de Sanidad Agraria, Ministerio de Agricultura (PNCBSENASA-MIAGRI), Lima, Perú.

Para la interpretación de los datos obtenidos se dividió a las evaluaciones semanales en cuatro periodos: Fase de putrefacción (fase fresca + fase de hinchazón) (Evaluación I = 17 de julio), fase de putrefacción activa (Evaluación del II al IV = 24 de julio al 07 de agosto), fase de putrefacción avanzada (Evaluación del V al VIII = 14 de agosto al 4 de septiembre) y fase de restos de esqueleto (Evaluación IX al XII = 11 de septiembre al 02 de octubre), tomando en consideración los cambios de descomposición físico-químicos ocurridos en el cadáver del "cerdo" (SCHoEnly 1992). Con la finalidad de evaluar la diversidad ecológica a nivel de especie (a) en las comunidades, fueron utilizados por muestreo los siguientes índices: ShannonWiener $\left(\mathrm{H}^{\prime}\right)$, Diversidad relativa, equitabilidad o Pielou $\left(\mathrm{J}^{\prime}\right)$ y dominancia Simpson (S) (Iannacone et al. 2000b, 2001).

Se empleó el coeficiente de correlación de Pearson entre las 12 evaluaciones semanales para relacionar a las especies necrófagas y depredadoras (ZAR 1996). Se empleó el índice cualitativo 2 de Kulcynski para determinar similaridad entre las evaluaciones semanales. Este índice se expreso como: $[1 / 2$ $(a+d / a+b+d+a+d / a+c+d)] \times 100$, donde: (a) número de taxas comunes en ambas evaluaciones, (b) número de taxas solo en una de las evaluaciones, (c) número de taxas solo en la otra evaluación, (d) número de taxas ausentes en ambas evaluaciones.

Consideramos como taxa individual a cada estadio de cada especie evaluada. Este índice varia entre 0 (ninguna sobreposición) a 1 (máxima sobreposición). Los taxas fueron agrupados, de acuerdo a esos valores, en un dendrograma, por el método de conglomerados UPGMA (unweighted pairgrouping group mean method). Se utilizó el paquete estadístico SPSS versión 10 para el cálculo de los estadísticos descriptivos e inferenciales.

\section{RESULTADOS Y DISCUSSION}

La tabla I nos muestra las ocho especies y el total de los 4405 especímenes colectados durante los 84 días de evaluación en el lechón del "cerdo". Tres de las especies fueron necrófagas: Cochliomyia macellaria (Fabricius, 1775) (Calliphoridae) (81,62\%); Dermestes maculatus (De Geer, 1774) (Dermestidae) $(16,35 \%)$ y Fannia canicularis (Linnaeus, 1761) (Muscidae) $(0,04 \%)$. Tres especies depredadoras: Saprinus aeneus (Fabricius, 1775) (Histeridae) (1,48\%); Necrobia rufipes (De Geer, 1775) (Cleridae) $(0,45 \%)$ y Hadruroides lunatus (L. Koch, 1867) (Iuridae) $(0,02 \%)$ y finalmente dos omnívoras: Linepithema humile (Mayr, 1868) (= Iridomyrmex humilis Emery, 1888) (Formicidae) (0,02\%); Porcellio laevis Latreille, 1804 (Porcellionidae) (0,02\%). Los insectos correspondieron al 99,96\% de los especimenes. Las formas inmaduras larvarias correspondieron al 76\%, las pupas al $14 \%$ y los adultos al $10 \%$ del total de especimenes colectados. Se observaron tres categorías ecológicas en la fauna cadavérica: necrófagos $(98,01 \%)$, depredadores $(1,95 \%)$ y omnívoros $(0,04 \%)$.

La tabla II indica las variaciones de la riqueza con un promedio de $2,25 \pm 1,21$ (1-5) y una abundancia de $365 \pm 684$ 
Tabla I. Artropofauna cadavérica asociada a Sus Scrofa en Ventanilla, Callao, Perú.

\begin{tabular}{|c|c|c|c|c|c|c|c|c|c|c|c|c|}
\hline Fecha de colecta & $17 / j u l$ & 24/jul & $31 / j u l$ & 07/ago & 14/ago & 21/ago & 28/ago & $04 /$ set & $11 /$ set & $18 /$ set & $25 /$ set & 02/oct \\
\hline Especies y fases de desarrollo & 1 & 2 & 3 & 4 & 5 & 6 & 7 & 8 & 9 & 10 & 11 & 12 \\
\hline \multicolumn{13}{|l|}{ Cochliomyia macellaria } \\
\hline L1 & 2030 & 0 & 0 & 0 & 0 & 0 & 0 & 0 & 0 & 0 & 0 & 0 \\
\hline L2 & 190 & 50 & 100 & 10 & 0 & 0 & 0 & 0 & 0 & 0 & 0 & 0 \\
\hline L3 & 130 & 150 & 160 & 130 & 0 & 0 & 0 & 0 & 0 & 0 & 0 & 0 \\
\hline Pupario & 100 & 0 & 280 & 40 & 70 & 0 & 0 & 0 & 0 & 0 & 0 & 0 \\
\hline Adulto (macho + hembra) & 25 & 0 & 80 & 10 & 0 & 40 & 0 & 0 & 0 & 0 & 0 & 0 \\
\hline \multicolumn{13}{|l|}{ Fannia canicularis } \\
\hline Pupa & 0 & 0 & 0 & 1 & 0 & 0 & 0 & 0 & 0 & 0 & 0 & 0 \\
\hline Adulto (hembra) & 0 & 0 & 0 & 1 & 0 & 0 & 0 & 0 & 0 & 0 & 0 & 0 \\
\hline \multicolumn{13}{|l|}{ Dermestes maculatus } \\
\hline L1 & 0 & 0 & 0 & 0 & 0 & 0 & 0 & 25 & 5 & 15 & 5 & 5 \\
\hline L2 & 0 & 0 & 0 & 0 & 5 & 5 & 35 & 10 & 20 & 10 & 100 & 30 \\
\hline L3 & 0 & 0 & 0 & 0 & 15 & 0 & 25 & 5 & 40 & 35 & 10 & 5 \\
\hline Pupa & 0 & 0 & 0 & 0 & 0 & 30 & 0 & 15 & 50 & 0 & 0 & 10 \\
\hline Adulto (macho + hembra) & 0 & 5 & 25 & 15 & 25 & 20 & 20 & 15 & 10 & 0 & 25 & 50 \\
\hline \multicolumn{13}{|l|}{ Saprinus aeneus } \\
\hline Adulto (macho + hembra) & 0 & 25 & 20 & 15 & 5 & 0 & 0 & 0 & 0 & 0 & 0 & 0 \\
\hline \multicolumn{13}{|l|}{ Necrobia rufipes } \\
\hline Adulto (macho + hembra) & 0 & 0 & 0 & 10 & 0 & 10 & 0 & 0 & 0 & 0 & 0 & 0 \\
\hline \multicolumn{13}{|l|}{ Linepithema humile } \\
\hline Adulto (hembra) & 0 & 0 & 0 & 0 & 0 & 0 & 0 & 0 & 1 & 0 & 0 & 0 \\
\hline Porcellio laevis (hembra) & 0 & 0 & 0 & 0 & 0 & 0 & 0 & 0 & 0 & 0 & 0 & 1 \\
\hline Hadruroides lunatus (hembra) & 0 & 0 & 0 & 0 & 0 & 0 & 0 & 0 & 0 & 0 & 0 & 1 \\
\hline
\end{tabular}

Fase fresca + fase de hinchazón = Evaluación 1; Fase de putrefacción activa = Evaluación 2 al 4; Fase de putrefacción avanzada = Evaluación 5 al 8; Fase de restos de esqueletos = Evaluación 9 al 12.

Tabla II. Riqueza, abundancia e Índices de diversidad de la artropofauna cadavérica en Sus scrofa en Ventanilla, Callao, Perú.

\begin{tabular}{|c|c|c|c|c|c|c|c|c|c|c|c|c|}
\hline Fecha de colecta & $17 /$ jul & 24/jul & $31 / j u l$ & 07/ago & 14/ago & $21 /$ ago & 28/ago & $04 /$ set & $11 /$ set & $18 /$ set & $25 /$ set & $02 /$ oct \\
\hline Parámetros & 1 & 2 & 3 & 4 & 5 & 6 & 7 & 8 & 9 & 10 & 11 & 12 \\
\hline Riqueza de especies & 1 & 3 & 3 & 5 & 3 & 3 & 1 & 1 & 2 & 1 & 1 & 3 \\
\hline Abundancia de especimenes & 2475 & 230 & 665 & 231 & 120 & 105 & 80 & 70 & 127 & 60 & 140 & 102 \\
\hline Índice de Diversidad (H') & 0 & 0,192 & 0,126 & 0,281 & 0,352 & 0,403 & 0 & 0 & 0,034 & 0 & 0 & 0,047 \\
\hline Índice de Pielou (J) & 0 & 0,402 & 0,264 & 0,466 & 0,737 & 0,844 & 0 & 0 & 0,071 & 0 & 0 & 0,098 \\
\hline Índice de Simpson (S) & 0 & 0.231 & 0.128 & 0.313 & 0.482 & 0.476 & 0 & 0 & 0.015 & 0 & 0 & 0.038 \\
\hline
\end{tabular}

Fase fresca + fase de hinchazón = Evaluación 1; Fase de putrefacción activa = Evaluación 2 al 4; Fase de putrefacción avanzada = Evaluación 5 al 8; Fase de restos de esqueletos = Evaluación 9 al 12. 
(50-2475). La riqueza más alta fue en el IV muestreo (fase de putrefacción activa). El índice de diversidad de ShannonWienner $\left(\mathrm{H}^{\prime}\right)$ fue de $0,119 \pm 0,151(0-0,403)$, el de Pielou $(\mathrm{J})$ fue de $0,240 \pm 0,305(0-0,844)$ y el de Simpson $(S)=0,140 \pm 0,188$ $(0-0,482)$. Los valores más altos de $\mathrm{H}^{\prime}$, J y $\mathrm{S}$ fueron en el VI muestreo (fase putrefacción avanzada) (Tab. II).

Durante el periodo de estudio la temperatura del aire fue de $17,70{ }^{\circ} \mathrm{C} \pm 1,73(15,08-20,05)$ y humedad relativa del aire fue $80,20 \% \pm 6,83(72,56-92,86)$ tomado de una estación meteorológica en la zona de estudio.

Las tres primeras fases presentaron mayores niveles de C. macellaria a nivel larval y pupal, $S$. aeneus y la abundancia total en comparación con las fases de putrefacción avanzada y restos de esqueletos. En contraste las formas inmaduras de $D$. maculatus fueron mucho mayores en la fase de restos de esqueletos.

El escarabajo depredador $S$. aeneus se encontró correlacionado positivamente $(r=0,81-0,82 ; \mathrm{p}=0,001)$ con la $\mathrm{L}_{2} \mathrm{y} \mathrm{L}_{3}$ de C. macellaria.

La figura 1 mostró alta similaridad para la artropofauna entre la fase fresca, fase de hinchazón y fase de putrefacción activa. Además, la fase de putrefacción avanzada y la fase de restos de esqueletos presentaron también, altas similaridades.

Los insectos son el grupo predominante durante la sucesión faunística. Los artrópodos necrófagos son considerados las más importantes especies que se alimentan de los tejidos cadavéricos (CATTs \& Goff 1992). En nuestro estudio se puede observar que en abundancia y en número de especies, los necrófagos son las más frecuentes (Tab. I).

Los Calliphoridae y los Dermestidae son los necrófagos con mayor importancia, siendo el primero más abundante durante la fase de putrefacción (fresca, hinchazón y putrefacción activa); en cambio los segundos incrementan durante la fase seca (putrefacción avanzada y restos de esqueletos) (Shoenly 1992, Lee 1996, Introna et al. 1997, Malgorn \& Coquoz 1999). Estas dos familias son las de mayor potencial forense. La primera ola de sucesión faunística está representada principalmente por la familia Calliphoridae. Esta familia es capaz de colonizar ambientes internos e inclusive acuáticos, principalmente durante las épocas de verano (PaYNe 1965; KeH 1985).

Diferentes especies de Calliphoridae alternan a lo largo del año en Buenos Aires, Argentina, influenciadas por la temperatura y el fotoperiodo (A. Oliva datos no publicados, 2000). ANDERSon (2000) indica que las especies de insectos y su secuencia de colonización en una carcasa animal, es impactada grandemente por la estación, hábitat, exposición a la luz solar y por la zona biogeoclimática evaluada. En la presente investigación, es sorprendente solo haber encontrado a una sola especie de Calliphorido, C. macellaria, y no a las especies Chrysomyia albiceps (Wiedemann, 1819), C. rufifacies (Macquart, 1850) y C. putoria (Wiedemann, 1830), ni tampoco a miembros de la familia Sarcophagidae. En un estudio preliminar, realizado en Lima, Perú en 1990 en carcasas de cerdos, se encontró a los calliphoridos C. albiceps, C. macellaria y a Lucilia sericata (Meigen, 1826) durante la época de verano (E. Barrientos y W. Dale comunicación personal). Quizás, los resultados obtenidos en este trabajo estén influenciados por el periodo de evaluación y al área geográfica, al estar restringidos a una sola época del año (invierno). Además, BRACK (1986) señala que el distrito de Ventanilla, Callao, Perú pertenece a la ecorregión del Desierto
Pacífico Costera, la cual presenta escasas precipitaciones y extrema aridez, y por lo tanto una muy pobre diversidad de flora y fauna, entre la que pudiera incluirse la artropofauna forense. Otro factor sería el efecto ocasionado por el fenómeno la Niña 1999-2000, posterior al evento el Niño 1997-98, la cual afectó a muchas poblaciones animales con relación a sus actividades biológicas (IANNACONE et al. 2000a), y posiblemente a las especies introducidas de género Chrysomyia. Godoy et al. (2001) han señalado que $C$. albiceps ha pesar de ser una especie con mayor habilidad competitiva que $C$. macellaria en condiciones experimentales y naturales, al mismo tiempo muestra una dinámica espacial y temporal más susceptible a los cambios ambientales. Además, Moura et al. (1997) señalan que las especies del género Chrysomyia prefieren grandes carcasas en comparación con pequeñas carcasas como la empleada en el presente estudio. Factores adicionales que explicarían el hecho que solo se encontrará una especie de calliphorido en el presente trabajo en comparación a otros trabajos de otras latitudes neotropicales con condiciones climáticas diferentes, sería la frecuencia de muestreo, en la que algunos autores señalan que debería ser diaria (CARVALHO et al. 2000), en especial al inicio del experimento; así como al hecho que solo se empleara un solo individuo de carcasa animal.

El Callifórido C. macellaria se le ha encontrado como una de las especies necrófagas más abundantes que colonizan carcasas animales terrestres (TOMBERLIN \& ADLER 1998), lo mismo ocurrió en este estudio. El poco número de especies encontradas en este trabajo en la carcasa de cerdo (Tab. I), está en contraste con otros trabajos (Hegazi et al. 1991, Shoenly 1992, Tomberlin \& ADLER 1998).

Smith (1986) indica que los grupos de artrópodos que siguen a los necrófagos en importancia, son los depredadores, como los Histeridae, Cleridae y el escorpión Hadruroides lunatus. En este estudio se les ha encontrado como los segundos en importancia después de los necrófagos (Tab. I).

SCHOENLy (1992) indica que en promedio la composición taxonómica es más pobre en especies al inicio y al final de los muestreos de sucesión faunística. Por eso al inicio y al final de todo el muestreo, se presentó la menor diversidad de $\mathrm{H}^{\prime}$, J y S (Tab. II). En cambio, a la mitad de todo el muestreo (VI) se presentó la mayor diversidad (Tab. II). Además la mayoría de la artropofauna cadavérica según Schoenly (1992) no es recurrente, es decir es persistente en la carcasa animal en un solo intervalo de tiempo, como se ha demostrado en el presente estudio (Tab. II y Fig. 1).

El empleo de un lechón de cerdo cercano a $4 \mathrm{~kg}$ se sustentó en los criterios propuestos por PAYNe (1965) y a la facilidad de manipulación de la carcasa para realizar un censo completo de la artropofauna. Algunos trabajos para evaluar entomofauna forense emplean carcasas de cerdo mucho mayores próximas a los $10 \mathrm{~kg}$ (CARvalHo et al. 2000) o cercanos a $23 \mathrm{~kg}$ (CATTs \& GofF 1992). Sin embargo algunos estudios que emplean cerdos con pesos bastante diferentes señalan que el tiempo de duración de las fases en descomposición varia, más no la estructura comunitaria de la artropofauna cadavérica (CATTS \& GofF 1992).

No se ha podido verificar en la presente investigación si la sustancia química empleada (succina-colina) en el sacrificio del cerdo perjudica la permanencia de la artropofauna en esta carcasa (CATTS \& Goff 1992). Siendo que muchos estudios prefieren sacrificar al cerdo empleado como carcasa en forma 


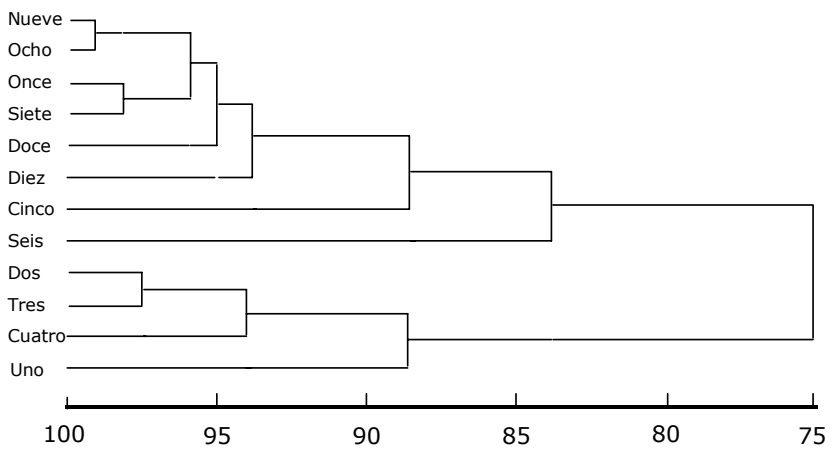

Fig. 1. Dendrograma de similaridad usando el Índice 2 de Kulcynski y posterior reescalamiento entre las doce evaluaciones semanales de la artropofauna en S. scrofa en Ventanilla, Callao, Perú. Uno: fase de putrefacción (Fase fresca + Fase de hinchazón); Dos al cuatro: fase de putrefacción activa; Cinco al ocho: fase de putrefacción avanzada; Nueve al Doce: fase de restos de esqueleto.

mecánica (CARVAlHo et al. 2000). Tampoco se ha corroborado si la abertura de la malla de la jaula empleada en esta investigación limita el acceso a algunas especies de artrópodos necrófagos.

La mayoría de estudios para evaluar la artropofauna cadavérica en cerdos utiliza protocolos con técnicas de colecta cualitativas (presencia o ausencia de determinados taxas) o semicuantitativas (Moura et al. 1997, CARvalHo et al. 2000), más no incluyen un análisis cuantitativo total de la fauna cadavérica presente por evaluación, como el reportado en el presente estudio. Sin embargo, como consecuencia de la colecta, determinación, conteo y posterior devolución de la mayoría de las formas inmaduras de insectos cadavéricos a la carcasa, se podría ocasionar aparentemente algunas imprecisiones desde el punto de metodológico, por el manipuleo de las formas inmaduras insectiles.

Cochliomyia macellaria (Calliphoridae) y D. maculatus (Dermestidae) son las dos especies de artrópodos con mayor potencial de uso forense en el Callao, Perú. Sin embargo, los resultados obtenidos a la fecha, muestran que es necesario evaluar en detalle la situación actual de la artropofauna asociada a carcasas animales en el Perú.

\section{AGRADECIMIENTOS}

A Amid Román, Priscilia Aguilar, Pilar García, Katia Álvarez \& Raúl Celis por su apoyo durante los censos de la artropofauna. A Alfonso Lizarraga e Isabel Bohórquez de la Universidad Nacional Federico Villarreal, Lima, Perú por la determinación de las especies de Coleóptera.

\section{REFERENCIAS BIBLIOGRÁFICAS}

Amendt, J.; R. Krettek; C. Niess; R. Zehner \& H. Bratzke. 2000. Forensic entomology in Germany. Forensic Science International, Limerick, 113: 309-314.

Amorin, J.A. \& O.B. Ribeiro. 2001. Distinction among the puparia of three blowfly species (Diptera: Calliphoridae) frequently found on unburied corpses. Memórias do Instituto Oswaldo Cruz, Rio de Janeiro, 96: 781-784.

Anderson, G.S. 2000. Establishing a countrywide database of insect succession on carrion in Canada. Abstract XXI International Congress of Entomology, XVIII Brazilian Congress of Entomology. Foz do Iguassu- Brazil 2000, August $20^{\text {th }}$ to $36^{\text {th }}$. Embrapa. p. 742.

BAUMGARTNER, D.L. 1993. Review of Chrysomyia rufifacies (Diptera: Calliphoridae). Journal of Medical Entomology, Lanham, 30: 338-352.

BeneCKE, M. 1998. Six forensic entomology cases: description and commentary. Journal of Forensic Sciences 43: 797805. Erratum: Journal of Forensic Sciences, Philadelphia, 46: 1303.

Barreto, M.; M.E. Burbano \& P. Barreto. 2002. Flies (Calliphoridae, Muscidae) and beetles (Silphidae) from human cadavers in Cali, Colombia. Memórias do Instituto Oswaldo Cruz, Rio de Janeiro 97: 137-138.

Borror, D.; D. DeLong \& C. Thriplehorn. 1989. An introduction to study of insects. New York, Saunders College Publishing, $6^{\text {th }}$ ed., $827 p$.

BRACK, E.A. 1986. Las ecorregiones del Perú. Boletín de Lima, Perú, 44: 57-70.

Byrd, J.H. \& J.F. ButLer. 1996. Effects of temperature on Cochliomyia macellaria (Diptera: Calliphoridae) development. Journal of Medical Entomology, Lanham, 33: 901-905.

Carvalho, L.M.; P.J. Thyssen; A.X. Linhares \& F.A. Palhares. 2000. A checklist of arthropods associated with pig carrion and human corpses in southeastern Brazil. Memórias do Instituto Oswaldo Cruz, Rio de Janeiro, 95: 135-138.

CatTs, E.O. \& M.L. Goff. 1992. Forensic entomology in criminal investigations. Annual Review of Entomology, Standford, 37: 253-272.

DALE, W.E. 1985. Identidad de las moscas Calliphoridae en la Costa Central del Perú. Revista Peruana de Entomología, Lima, 28: 63-70.

Dear, J.P. 1985. A revision of the New World Chrysomini (Diptera: Calliphoridae). Revista Brasileira de Zoologia, Curitiba, 3: 109-169.

Faria, L.B. \& W.A.C. Godoy. 2001. Prey choice by facultative predator larvae of Chrysomya albiceps (Diptera: Calliphoridae). Memórias do Instituto Oswaldo Cruz, Rio de Janeiro 96: 875-878.

Godoy, W.A.C.; F.J. von Zuben; C.J. von Zuben \& S.F. Reis. 2001. Spatio-temporal dynamics and transition from Asymptotic equilibrium to bounded oscillations in Chrysomya albiceps (Diptera: Calliphoridae). Memórias do Instituto Oswaldo Cruz, Rio de Janeiro, 96: 627-634.

GREenBERG, B. \& M.L. SzYSKA. 1984. Immature stages and biology of fifteen species of Peruvian Calliphoridae (Diptera). Annals of the Entomological Society of America, Lanham, 77: 488-517.

Hegazi, E.M.; M.A. ShaAban \& E. Sabry. 1991. Carrion insects of the Egyptian western desert. Journal of Medical Entomology, Lanham, 28: 734-739.

Iannacone, J.; L. Alvariño; R. Moreno; M. Reyes \& J. Chauca. 2000 a. Culícidos (Diptera) del río Chillón y sectores adyacentes de la provincia Constitucional del Callao, Perú, durante el Niño 1997-1998. Acta Entomológica Chilena, Santiago de Chi- 
le, 24: 51-60.

Iannacone, J.; M. Alayo; J. Sánchez. 2000b. Biodiversidad de la artropofauna del Bosque Zárate, Lima, Perú, empleando tres técnicas de censo. Wiñay Yachay, Lima, 4 (1): 27-46.

Iannacone, J.; M. Alayo; A. Arrascue; J. Sánchez \& M. Abanto. 2001. Las trampas de luz para evaluaciones rápidas de la biodiversidad de la artropofauna: análisis de tres casos. Wiñay Yachay, Lima, 5: 7-20.

Introna, F.JR.; C.P. CAmpobasso \& A. DI Fazio. 1998. Three case studies in forensic entomology from southern Italy. Journal of Forensic Sciences, Philadelphia, 43: 210-214.

Кен, B. 1985. Scope and applications of Forensic Entomology. Annual Review of Entomology, Standford, 30: 137-154.

Komar, D. \& P. Beattie. 1998. Postmortem insect activity may mimic perimortem sexual assault clothing patterns. Journal of Forensic Sciences, Philadelphia, 43: 792-796.

LeE, H.L. 1996. Recovery of forensically important insect larvae from human cadavers in Malaysia. Malaysian Journal of Pathology, Kuala Lumpur, 18: 125-127.

Liu, D. \& B. Greenburg. 1989. Immature stages of some flies of forensic importance. Annals of the Entomological Society of America, Lanham, 82: 80-93.

Malgorn, Y. \& R. Coquoz. 1999. DNA typing for identification of some species of Calliphoridae. An interest in forensic entomology. Forensic Science International, Limerick, 02: 111-119.

Moura, M.O.; C.J.B. Carvalho \& E.L.A. Monteiro-Filho. 1997. A preliminary analysis of insects of medico-legal importance in Curitiba, State of Paraná. Memórias do Instituto Oswaldo Cruz, Rio de Janeiro, 92: 269-274.

Payne, J.A. 1965. A summer carrion study of the baby pig Sus scrofa Linnaeus. Ecology, Tempe, 46: 592-602.

PeÑa, L.E.G. 1992. Introducción a los insectos de Chile. Ed. Universitaria ( $3^{\text {er }}$ Ed.). Santiago, Chile. 256 pp.

SCHoenty, K. 1992. A statistical analysis of successional patterns in carrion-arthropod assemblages: implications for forensic entomology and determination of the postmortem interval. Journal of Forensic Sciences, Philadelphia, 37: 1489-1513.

Smith, K.G.V. 1986. A Manual of Forensic Entomology. New York, Comstock, 205p.

Solervicens, J. 2001. Clave para géneros de Cleridae de Chile (Coleoptera). Acta Entomológica Chilena, Santiago de Chile, 25: 41-46.

SouZA, A.M. \& A.X. Linhares. 1997. Diptera and coleoptera of potential forensic importance in southeastern Brazil: Relative abundance and seasonality. Medical and Veterinary Entomology, London, 11: 8-12.

SтоскwELL, S.A. 1992. Systematics observations on North American Scorpionida with a key and checklist of the families and genera. Journal of Medical Entomology, Lanham, 29: 407-422.

TANTAWI, T.L. \& E.M. EL KaDy. 1997. Identification of third instar larvae of forensically important flies (Diptera: Calliphoridae, Sarcophagidae and Muscidae) in Alexandria, Egypt. The Journal of the Egyptian-German Society of Zoology, Cairo, 23: 1-20.

Tomberlin, J.K. \& P.H. Adler. 1998. Seasonal colonization and decomposition of rat carrion in water and on land in an open field in South Carolina. Journal of Medical Entomology, Lanham, 35: 704-709.

VAN LAerhoven, S.L. \& G.S. ANDERson. 1999. Insect succession on buried carrion in two biogeoclimatic zones of British Columbia. Journal of Forensic Sciences, Philadelphia, 44: 32-43.

von Zuben, C.J.; R.C. Bassanezi \& F.J. Zuben. 1998. Theoretical approaches to forensic entomology: II. Mathematical model of larval development. Journal of Applied Entomology, Berlin, 122: 275-278.

ZAR, J.H. 1996. Biostatistical Analysis. New Yersey, PrenticeHall. Inc., $3^{\text {th }}$ Ed., 662p.

Recebido em 30.IX.2002; aceito em 27.II.2003. 